\title{
Oeber eine Bemerkung des Hrn. Dr. K. Kisskalt betreffs einer Arbeit über den Bacillus prodigiosus.
}

$$
\text { Von }
$$

\author{
Dr. E. Bertarelli,
}

Priratdocent in Turin.

Im II. Theile seiner interessanten Arbeit „Beiträge zur Lehre von der natürlichen Immunität" I bat Dr. Kisskalt eine Studie von mir „Untersuchungen und Beobachtungen über Biologie und Pathogenität des B. prodigiosus" ${ }^{2}$ citirt und dazu verschiedene Bemerkungen gegeben, die mich zu einer einfachen Erklärung veranlassen.

Kisskalt sagt S. 256: „Es sei hier noch erwähnt, dass Bertarelli eine Vermehrung des Prodigiosus im Thierkörper nachgewiesen zu haben glaubt. Aber abgesehen daron, dass er zur Tödtung der Thiere grosse Dosen nöthig hatte, scheint der Mikroorganismus, mit dem er arbeitete, gar kein Prodigiosus gewesen zu sein, da er eine Erhitzung auf $80^{\circ}$ 1 Stunde lang aushielt, also Sporen bildete, eine Eigenschaft, die am echten Prodigiosus bisher niemals beobachtet wurde."

Nun muss ich vor Allem bemerken, dass der Keim, mit dem ich gearbeitet habe, ein typischer Prodigiosus ist, der bezüglich aller charakteristischen Eigenschaften mit anderen Prodigiosusbacillen verschiedenster Abstammung übereinstimmte. Auch habe ich niemals behauptet, dass dieser Keim Sporen besitze. Ich habe wohl geschrieben, dass: „die durch Hitze abgetödteten Bouillonculturen (der Prodigiosus ist sehr widerstandsfähig, seine nach zahlreichen Seriendurchgängen erhaltenen Culturen widerstehen noch 1 Stunde lang bei selbst $80^{\circ}$ )..." wozu ich bemerke, dass ich natürlich von der Resistenz der Culturmasse und nicht des Keimes an und für sich reden wollte. Lässt man nun in einem Wasserbad auf

I Diese Zeitschrift. Bd. XIVII.

2 Centralblatt für Bakteriologie. Abth. I. Bd. XXXIV. 


\section{E. Bertareitil: Über eine Bemerkung des Hrin. Dr. Kisskalt.}

$80^{\circ}$ Bouillonculturen dieses Prodigiosus, so tritt wirklich der Fall ein, dass nach 1 Stunde nicht alle Culturen ganz steril sind. Das leuchtet ein, da man weiss, dass die Hitze in solchen Verhältnissen sich schwer im Milieu verbreiten kann, und dass bei eventuellen Keimanhäufungen die in der Mitte liegenden Keime von der Einwirkung der Hitze durch die umliegenden Keime der Anhäufung geschützt werden.

Nun wollte ich mit meinen Worten nur sagen: dass mein Prodigiosus äusserst resistent war, derart, dass die massenweise auf $80^{\circ}$ in ein Wasserbad gebrachten Bouillonculturen nach 1 Stunde nicht immer steril ausfielen. Diese Thatsache darf diejenigen nicht besonders wundern, die beobachtet haben, wie seltene Male Bouillonculturen wenig resistenter Keime, wie die Choleravibrio auf $60^{\circ}$ im Wasserbad nicht absolut steril sind, auch selbst nach relativ langer Zeit. Von hier bis zur Behauptung, dass der in dünnen Schichten liegende Keim (auf gewöhnlichen Seidenfäden oder Deckgläschenstückchen) resistent sei, ist noch ein weiter Weg. Unter derartigen Verhältnissen stirbt mein Prodigiosus auch bei $60^{\circ}-$ damit fällt die Interpretation und Deduction, die Kisskalt in gutem Glauben der Thatsache gegeben hat. Was übrigens die Frage der Beziehungen zwischen $\mathrm{B}$. prodigiosus und natürlicher Immunitätslehre anbetrifft, darauf hoffe ich später mit experimentellen Daten zurückkommen zu können. Uebrigens sind in jedem Falle die Culturen auf Verlangen zur Verfügung; es sei mir ausserdem gestattet beizufügen, dass nicht nur einige der von mir studirten Thatsachen in meiner Arbeit über Prodigiosus mit der Ansicht A. Dettweiler's ${ }^{1}$ (die zu einem anderen Zwecke gemacht und auf anderen Wegen) übereinstimmen, sondern auch das eventuelle pathogene Vermögen des Prodigiosus - kürzlichst auch von Lombardo Pellegrino ${ }^{2}$ im Laboratorium des Prof. Sanfelice mit. einem direct aus dem Terrain isolirten Prodigiosus - bestätigt wurde.

1 Coll. Transact. of the Assoc. of Americ. Physic. 1903. Vol. V.

3 Giorn. d. R. Soc. Ital. d'Igiene. 1904. Hft. 1. 